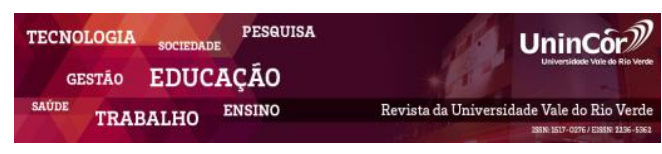

Revista da Universidade Vale do Rio Verde ISSN: 1517-0276 / EISSN: 2236-5362 v. $17 \mid$ n. 1 | Ano 2019

Wellington Fernando da Silva Ferreira Centro Universitário Campos de Andrade UNIANDRADE wellingtonferreira42@gmail.com

Elia Machado de Oliveira Universidade Estácio de Sá - ESTÁCIO elia561@ hotmail.com

\section{ENSINO A DISTÂNCIA (EAD): TÁTICAS \\ NA IMPLANTAÇÃO DE CARGA HORÁRIA EM 20\% NAS UNIVERSIDADES}

\section{RESUMO}

Introdução: A educação a distância (EAD) abre-se a um paralelo, a novas alternativas de ensino, qual a implementação do sistema de metodologia a distância, emerge a necessidade de realização das disciplinas em $20 \%$ de sua carga horaria, nas instituições de ensino, para tal, evidencia-se, o questionamento a serem desvelados. Objetivo: Analisar a estruturação do ensino à distância, e a importância da implantação da modalidade à distância no ensino superior em saúde, descritas nas publicações cientificas. Metodologia: Trata-se de um estudo de revisão sistemática, transversal, exploratório, com abordagem qualitativa, realizou-se a busca de estudos em periódicos nacionais: SCIELO, LILACS, BVS, publicados entre os anos 2013 ao primeiro semestre de 2017, com amostra final constituída por 14 artigos científicos completos. Resultados e discussões: Os achados apontam uma produção atual escassa nas dimensões proposta desta temática, em linhas gerais ressaltaramse trabalhos científicos voltados a eixos fundamentais; Contexto Histórico do EAD no Brasil, Organizando as estruturas nas instituições de ensino à cerca do EAD Importância da modalidade EAD para o ensino Superior, Implementação dos $20 \%$ nas disciplinas no ensino superior e Capacitação do Tutor, os estudos concentra-se em todas as regiões do pais. Conclusão: A reflexão e compreensão dos aspectos de importância da implantação da modalidade à distância no ensino superior em saúde e como são selecionadas as disciplinas à distância $20 \%$ EAD nas Instituições de ensino estimulando e evidenciando as estratégias, propostos e arquitetados como objetivos foram alcançados, e seus resultados, através desta investigação apresentam relevância social, profissional e acadêmica.

Palavras-chave: Educação a Distância; Capacitação Docente; Tecnologias Na Educação em Saúde.

\section{TEACHING AT DISTANCE (EAD): TACTICS IN THE LOADING OF TIME LOADING BY 20\% IN UNIVERSITIES}

\title{
ABSTRACT
}

Introduction: Distance education (ODL) opens up a parallel, new teaching alternatives, how to implement the distance learning methodology, the need to perform the subjects in $20 \%$ of their workload, in the institutions of teaching, for this, it is evident, the questioning to be unveiled. Objective: To analyze the structure of distance education, and the importance of implementing the distance modality in higher education in health, described in scientific publications. Methodology: This is a systematic, cross-sectional, exploratory study with a qualitative 
approach. The study was carried out in periodicals: SCIELO, LILACS, VHL, published between the years 2013 and the first semester of 2017, with a final sample consisting of 14 complete scientific articles. Results and discussions: The findings point to a scarce current production in the proposed dimensions of this theme, in general lines emphasized scientific works focused on fundamental axes; Historical Context of the EAD in Brazil, Organizing the structures in the educational institutions around the EAD Importance of the modality EAD for Higher Education, Implementation of the $20 \%$ in the disciplines in higher education and Tutor Training, studies are concentrated in all regions from the country. Conclusion: The reflection and understanding of the importance aspects of the implementation of the distance modality in the higher education in health and how the disciplines at a distance distance $20 \%$ are selected in the teaching institutions stimulating and evidencing the strategies, proposed and architected as objectives were reached, and their results, through this research, have social, professional and academic relevance.

Keywords: Distance Education; Teacher Training; Technologies in Health Education.

Recebido em: 05/08/2018 - Aprovado em: 03/04/2019 - Disponibilizado em: 15/07/2019

\section{INTRODUÇÂO}

A tecnologia em seu escopo propõe diversas faces à contemporaneidade, o acesso a informação vem reformulando todas as áreas do conhecimento/ensino, quebrando paradigmas com a implantação das Tecnologias da Informação e Comunicação (TIC) (LINO, et al. 2013). Embora seja algo que surgiu em meados de 1970 durante a revolução industrial, e cada vez mais esta sendo discutida e inserida em instituições de ensino somando-se a diferentes aspectos oriundos do avanço educação (CERDAS, et al. 2013; MANGAN, et al. 2013; JUNIOR e FERNANDES 2014; SÁ, 2015).

Estas novas mudanças fazem parte do mundo contemporâneo, fazendo parte do dia a dia de pessoas, seja no trabalho, laser ou em instituições de ensino (MENESES, et al. 2016;
CARVALHO, 2017). Contudo à uma preocupação com a qualidade e a forma que será sistematizada para que o aprendizado não perca a qualidade cognitiva, e não torne-se transigente, mas um processo de informação as instituições de ensino, e potencializando os saber em cada esfera do conhecimento (ARAÚJO, 2014; SEGENREICH， 2014; SEBASTIÃO， 2015; MAIA, 2017).

No que tange a educação em torno de seu pilares como aprender para toda vida, ressalta-se, o aprender a fazer por meio de competências e vivencia de situações, aprender gerenciando conflitos no qual desenvolve discernimento do conhecimento e aprender a ser com capacidade de autonomia e fortalecimento do caráter. Contudo aprender sempre necessita de uma integração de atividades bem elaboradas e fundamentadas por meio do projeto político pedagógico 
(LUVIZOTTO e CARNIEL, 2014; RIBEIRO e SOUZA, 2016; CAETANO, et al. 2016; CARVALHO, 2017).

Neste sentido da educação, abre-se um paralelo a novas alternativas de ensino, qual a implementação do sistema de metodologia a distância, emerge a necessidade de realização das disciplinas em $20 \%$ de sua carga horaria, nas instituições de ensino, qual requer uma reflexão crítica e ao mesmo tempo criativa assegurando que estes pilares não se transformem em montante de formação desnecessário e sim em ferramenta de qualidade para auxílio na educação e aos que utilizam deste métodos, perpassando um efetivo aproveitamento das informações e não meros receptores das mesmas (OLIVEIRA, 2010; MOMO e BEHR, 2016; MARCUZZO, et al. 2017).

Embora as disciplinas 20\% apresentarem uma flexibilidade de acesso aos conteúdos de ensino e aprendizagem programados pela matriz curricular com disciplinas especificas, há, para tal, um regulamento a ser seguido conforme a resolução PORTARIA $\mathrm{N}^{\circ}$ 4.059, DE $10 \mathrm{DE}$ DEZEMBRO DE 2004 (DOU de 13/12/2004, Seção 1, p. 34).

Está implantação conforme legislação, requer que no projeto político pedagógico e na matriz curricular, esteja de forma integrada e assistida de uma estratégia significativa como ambientes de aprendizagem por meio das tecnologias e distribuição de conteúdo acerca dos objetivos pedagógicos (MANGAN, et al. 2013).

Todavia o ensino presencial e à distância no qual tange a diversidade de ofertas integradas, quais estão amparando-se a muitas instituições de ensino e corroboram para muitos pesquisadores esta nova metodologia de ensino (JUNIOR, 2009; JUNIOR e FERNANDES 2014; SÁ, 2015; CAETANO, et al. 2016).

Por tanto se faz necessário um sistema solido para contribuir ao acesso de todos os envolvidos socializando e propiciando algo a mais aqueles que nunca tiveram acesso a este modelo de educação. Com esta perspectiva deste novo métodos implantado na modalidade dos $20 \%$ EAD todos terão a oportunidade do acesso (OLIVEIRA, 2010; CERDAS, et al. 2013; LINO, et al. 2013; ARAÚJO, 2014 MARCUZZO, et al. 2017).

Neste sentido conforme justifica-se a problemática supracitada, o presente estudo, tem como objetivo analisar a estruturação do ensino à distância, e a importância da implantação da modalidade à distância no ensino superior e como são selecionadas as disciplinas à distância $20 \%$ EAD em saúde nas Instituições de ensino.

\section{MÉTODOS}

Trata-se de um estudo de revisão sistemática, transversal, exploratório, com abordagem qualitativa. Esse tipo de pesquisa objetiva desvendar, recolher e analisar principais contribuições teóricas sobre um determinado fato, recurso importante da prática baseada em evidências, que consiste em uma forma de síntese dos resultados de pesquisas relacionados com um problema específico (NASCIMENTO e TEIXEIRA, 2012).

Para obtenção dos artigos explorados, utilizou-se o descritor em ciências da saúde 
(DeCS): "Ensino a Distância (EAD)", em português na Scientific Eletronic Library Online (SCIELO).

O período selecionado para a pesquisa foi de 2013 a primeiro semestre de 2017, com idioma em português. O recorte temporal do estudo é justificado por possibilitar o acesso aos dados de recentes investigações. Após a seleção primária dos artigos, foram analisados os títulos e resumos, levando-se em conta os critérios de inclusão.

Os critérios de inclusão foram textos brasileiros. As categorias escolhidas para os estudos foram: 1. Base de dados; 2. Assunto principal; 3. Limites humanos; 4. País /Região; 5. Idioma; 6. Recorte temporal; 7. Amostra final.

Foram utilizados os seguintes critérios de exclusão: Manuscritos quais não versaram sobre ensino a distância EAD e sua aplicabilidade de carga horaria em $20 \%$ e que não tinham open access.

Contudo, alguns artigos não foram incluídos, devido à duplicidade encontrada nas diferentes bases consultadas. Dessa forma, na última fase de seleção, realizada a leitura integral de todos os artigos, a amostra final foi constituída por quatorze (14) artigos completos.

Os artigos receberam leitura exploratória e analítica, com a finalidade de identificar a validade do documento para o presente trabalho; em seguida, resumido as informações sobre o objeto de estudo para posteriormente, receberem uma leitura de característica interpretativa, com o problema proposto, foram considerados aspectos qualitativos, focando a discussões sobre a temática.
O presente estudo traz a seguinte questão norteadora: Quais são as implantações da modalidade à distância e as disciplinas à distância $20 \%$ EAD nas Instituições de ensino?.

\section{RESULTADOS E DISCUSSÃO}

\subsection{Argumento Histórico do EAD no Brasil}

A introdução da Educação à Distância EAD, no sistema educacional brasileiro, deu-se a partir da promulgação da Lei no 9.394/96 - Lei de Diretrizes e Bases na Educação, mas com outra forma de expressão de sua nomenclatura, sendo designada inicialmente como Ensino à Distância EAD. Nos artigos 80 e 81 da respectiva lei, podese encontrar as fundamentações especificas no que tange a viabilização e organização das atividades de ensino e aprendizagem frente as instituições de ensino (MANGAN, et al. 2013; JUNIOR e FERNANDES 2014; LUVIZOTTO e CARNIEL, 2014; RIBEIRO e SOUZA, 2016).

$\mathrm{Na}$ sequência, através da Portaria $\mathrm{n}^{\circ}$ 2.253/2001, o Ministério de Educação e Cultura, determinou que fossem devidamente informadas à SESU/MEC todas as modificações efetuadas pelas Instituições de Ensino, em seus projetos pedagógicos balizadores dos cursos de graduação já reconhecidos, buscando unicamente uma adequação de oferta das disciplinas semipresenciais.

Em 10 de dezembro de 2004, através da Portaria MEC no 4.059, ocorreu a revogação da Portaria anterior $\mathrm{n}^{\circ}$ 2.253/2001, mas não alterando em nada a possibilidade de implantação de disciplinas semipresenciais nas matrizes 
curriculares dos cursos presenciais (MANGAN, et al. 2013; JUNIOR e FERNANDES, 2014).

Frente a manutenção da legalidade quanto à implantação das disciplinas semipresenciais, elencou-se o entendimento sobre qual a correta identificação das disciplinas com possibilidade de adequação frente a nova metodologia, reconhecendo-se as mesmas como atividades didático-pedagógicas, que são ministradas com a utilização de tecnologias de comunicação remota, com diversos suportes de informação (LUVIZOTTO e CARNIEL, 2014; RIBEIRO e SOUZA, 2016; MARCUZZO, et al. 2017).

No artigo $2^{\circ}$ da Portaria $n^{\circ} 4.059$, encontra-se a fundamentação necessária para aplicação dos procedimentos a serem adotados, frente a possibilidade de transferência de $20 \%$ do total previsto de carga horária em cursos de graduação presencial para a plataforma de Educação a Distância - EAD (SEGENREICH, 2014; SEBASTIÃO, 2015).

Com o surgimento das novas diretrizes, surgiram também questionamentos quanto a correta conceitualização de Ensino à Distância, sendo que através do Parecer CNE/CES no 63/2003, observou-se que a expressão mais apropriada a ser aplicada seria Educação à Distância, utilizando-se da mesma até os dias atuais (MANGAN, et al. 2013; JUNIOR e FERNANDES, 2014; MARCUZZO, et al. 2017).

\subsection{Aparelhando as composições nas instituições de ensino à cerca do EAD}

Em linhas gerais a responsabilidade no trato à EAD, cabe as instituições de ensino, desenvolverem estratégias alicerçadas nas legislações e na matriz didática pedagógica. Para tal, baseia-se na necessidade da implementação de uma conjuntura do modelo pedagógico destinado ao EAD, haja vista a necessidade de adaptação, capacitação e planejamento no desenvolvimento dos docentes envolvidos acerca da modalidade (LIMA, et al. 2014; LUVIZOTTO e CARNIEL, 2014; RIBEIRO e SOUZA, 2016).

Complementando a necessidade de criação de uma metodologia de ensino direcionadas aos discentes e docentes minimizando as falhas no decorrer do processo pedagógico devem construídas e pautadas na legislação. Por meio da estruturação do Ambiente Virtual de Aprendizagem (AVA) permitindo que ocorra a interação proposta pelo EAD na qual haverá a mediação no ensino, contando com apoio de uma equipe especializada para manutenção do sistema para garantir um bom andamento e qualidade do serviço (FRANZIN, et al. 2015; AHAD, et al. 2017).

Contudo para que se tenha um efetivo andamento, necessita-se de um a equipe técnica para dar suporte aos sistema interligando as informações com o corpo pedagógico no planejamento conforme as diretrizes solicitadas no ensino EAD. As informações geram de forma rápida atraindo atenção de gestores sobre a importância de um suporte adequado para assegurar significativamente uma qualidade do ensino EAD (LUVIZOTTO e CARNIEL, 2014; RIBEIRO e SOUZA, 2016).

Cabe a parte técnica do ambiente virtual criar uma estrutura plena, que garanta o andamento das instalações necessária para manter uma atividade educativa a distância com 
qualidade e um planejamento conduzindo conforme pede o modelo pedagógico do ensino a distância. Contudo autores relatam sobre a importância de se ter um suporte técnico coerente e que atenda todas as necessidades condizentes e potencializando o ensino a distância (SEGENREICH, 2014; SEBASTIÃO, 2015).

Tais conjunturas também exige que a organização projeto político pedagógico da instituição e gestores tenha uma atenção para que o curso não perca a essência da qualidade perante comissões avaliadoras por meio do exame Nacional de desempenho de Estudantes (ENADE), Sistema Nacional de Avaliação da Educação Superior (SINAES) e o Instituto Nacional de Estudos e Pesquisas Educacionais (INEP). Neste sentido na maioria das instituições conta com uma equipe qualificada para o ensino a distância estruturada para nova modalidade a cerca de um modelo pedagógico (MANGAN, et al. 2013; JUNIOR e FERNANDES 2014; FRANZIN, et al. 2015; AHAD, et al. 2017).

Portanto, investir em capacitação de tutores permanentes prevê que a estrutura seja significativa para o suporte didático pedagógico, capaz de dar uma acessória aos discentes com rotinas de orientações fundada em uma pratica pedagógica. Acredita-se que para uma excelente desenvoltura em ensino a distância há necessidade de um suporte técnico com qualidade para postagem de matérias, interação no sistema e a integração do aluno (FRANZIN, et al. 2015; MARCUZZO, et al. 2017).

\subsection{Seriedade da modalidade EAD para o ensino superior}

No modelo de ensino a distância nas instituições de ensino superior, percebe-se a necessidade de maior, transmissão de conhecimento tendo por objetivo por meio da tecnologia garantir um aprendizado eficiente que constrói e prepara alunos para obtenha por meio do conhecimento as competência e habilidades necessárias para o mercado de trabalho. Este modelo de ensino nas instituições se preparam para trabalhar de forma multilateral, no qual o aluno interage com o seguimento de tutoria e atividades em um ambiente virtual integrado (LIMA, et al. 2014; LUVIZOTTO e CARNIEL, 2014; RIBEIRO e SOUZA, 2016).

Por mais que o aluno não se encontre no mesmo espaço físico com a tutoria a instituição não se exime de sua responsabilidade de oferecer um ensino com qualidade. A tutoria é um mediador ao aluno durante sua aprendizagem que é um dos grandes cuidados de gestores que implantam esta modalidade em suas instituições de ensino (MANGAN, et al. 2013; JUNIOR e FERNANDES 2014).

Trabalhos mencionam que o ensino a distância teve uma aumento expressivo no Brasil segundo último censo realizado pela Associação Brasileira de Educação a distância 226 instituições de ensino realizam esta modalidade, sendo destas $64 \%$ são de instituições privadas. Segundo estudos à o número de solicitações de credenciamento nesta modalidade inclusive instituições consideradas as mais tradicionais estão optando por instituir ensino a distância (SEGENREICH， 2014; SEBASTIÃO， 2015; MARCUZZO, et al. 2017). 
Contudo uma das maiores preocupação do ensino a distância é manter a motivação e o interesse do aluno pelo método de aprendizagem levando a gestores a repensar no que tange a transmissão de conhecimento, entre construir um conhecimento firmado em métodos pedagógicos eficientes (MANGAN, et al. 2013; JUNIOR e FERNANDES 2014; FRANZIN, et al. 2015; AHAD, et al. 2017).

\subsection{Implementação dos $20 \%$ nas disciplinas no ensino superior}

Por meio da portaria $2.253 \mathrm{de}$ 18/10/2001 fui instituído início somente à instituições de ensino do sistema Federal instituam em seu projeto pedagógico e curricular disciplinas no modalidade a distância não excedendo os $20 \%$ da carga horária total do curso (SIMÕES, 2016).

\section{O Ministério da Educação (MEC)} juntamente com o Sistema de Seleção Unificada (SESU) irão gerenciar a qualidade do ensino a distância. Fica acordado que as atividades serão online, com tutores capacitados mantendo um ensino de qualidade. Contudo está portaria não deixa claro se os $20 \%$ do ensino a distância engloba todas as disciplinas ou seria $20 \%$ para cada disciplina observada por muitos autores (LIMA, et al. 2014. SIMÕES, 2016; MARCUZZO, et al. 2017).

Diferente da portaria 4.059 que deixa claro as regras a ser tomada por cada instituição que se encontre regularizada diante o MEC sobre a forma que devem distribuir suas disciplinas e as forma de avaliação. Nesta continuidade deve se repensar na mediação do conhecimento por meio de um bom sistema tecnológico que possibilite que os recursos didáticos pedagógicos seja efetivamente repassados na integra, possibilitando o auto aprendizagem. Uma das grandes preocupações de muitos autores não é com a tecnologia em si, pois a mesma serve como instrumento de acesso, entretanto a conformação em que estes conteúdos são transmitidos para um aprender e ensinar efetivo (LUVIZOTTO e CARNIEL, 2014; FRANZIN, et al. 2015; RIBEIRO e SOUZA, 2016; AHAD, et al. 2017).

Portanto fazer uma análise do projeto pedagógico regularmente é a chave principal para o sucesso e identificar pontos positivos e negativos propiciando readequações no que for necessário (SIMÕES, 2016). Entretanto no processo de aprendizagem e no desenvolvimento do docente e discente observa-se desafios acerca do ensino a distância, até sua devida implantação e sua rotina estabelecida (FRANZIN, et al. 2015; AHAD, et al. 2017).

Diversos autores salientam em seus estudos, que é de extrema relevância o preparo, capacitação e a qualificação dos docentes quanto a dinâmica do processo do ensino a distância e utilização do ambiente virtual ou outras plataformas, não somente de modo obrigatório mas com finalidades de aprendizagem e novos conhecimentos (MANGAN, et al. 2013; LIMA, et al. 2014; JUNIOR e FERNANDES 2014).

Outros fatores evidenciados em estudos apontam a viabilidade da utilização de $20 \%$ da carga horária nas disciplinas semi-presenciais, destaca-se ainda a existência de características do participante do ensino a distância, no qual o docente tutor, apresenta o perfil muitas vezes da 
área tecnológica porem sem a vivencia das práticas pedagógicas no ensino a distância (MARCUZZO, et al. 2017).

\subsection{Capacitação do tutor}

Se faz necessário retroceder um pouco no tempo, mais precisamente no meados do século XV quando a tutoria já se fazia presente em universidades na qual atuação de orientação à estudos religiosos que posteriormente assume o papel de orientador em trabalhos acadêmicos com o mesmo objetivo apresenta-se nos dias atuais como tutores em ensino a distância (SIMÕES, 2016; MARCUZZO, et al. 2017).

Para compreensão fundamentada do papel de Tutoria são profissionais da educação qualificados tendo a função de mediador do conhecimento por meio online a distância e a integração com pratica pedagógica, o saber e o aprendiz (FRANZIN, et al. 2015; AHAD, et al. 2017).

A tutoria é considerada um dos pilares fundamentais para desenvoltura dos acadêmicos, contudo esta demanda não cabe somente ao professor tutor e sim a todos os envolvidos, Segundo a Lei Federal 10.172, de 09 de janeiro de 2001 que aprova o ensino a distância no Brasil, que prevê nesta resolução a necessidade de capacitação continuada destes professores Tutores para as disciplinas em EAD (SEGENREICH, 2014; SEBASTIÃO, 2015).

É importante ressaltar que o tutor é um diferencial no ensino a distância por meio da mediação e a construção do conhecimentopor meio de suas atividades por meio da orientação aos acadêmico e averiguar se a aprendizagem está atingindo os objetivos, também cabe a responsabilidade da organização de planos de ensino e a produção de matérias por meio de pesquisas para que se tenha um material atualizado (LUVIZOTTO e CARNIEL, 2014; RIBEIRO e SOUZA, 2016).

Outras atividades que podemos destacar, não menos importante é a orientação para acadêmicos caso haja dúvidas sobre conteúdo e manter uma organização lógica de conteúdos pedagógicos adequado a disciplina. A tutoria vai além das organizações é um caminho trilhado no aprender a aprender, no qual o incentivo ao aluno percorre ao longo do curso (SEGENREICH, 2014; SEBASTIÃO, 2015).

O EAD demanda dos tutores uma organização prévia seja qual for a instituição de ensino, para que haja uma constante analise do desenvolvimento das atividades e avaliações e um constante seguimento da aprendizagem do alunos, a formação de profissionais para a tutoria requer conhecimento e dedicação por parte de quem ensina e de quem aprende (MANGAN, et al. 2013; JUNIOR e FERNANDES, 2014; FRANZIN, et al. 2015; SIMÕES, 2016; MARCUZZO, et al. 2017; AHAD, et al. 2017).

\section{CONCLUSÃO}

A educação consecutivamente tratou do preparo dos sujeitos para seu papel na sociedade, a primazia da educação formal decaiu por causa do surgimento de uma aprendizagem que se estende por toda a vida e da educação informal, assim como de novas áreas de aprendizagem. Configurações de treinamento feitas no emprego, 
o desenvolvimento pessoal de planos e de cursos de competência para novos gerentes tornaram-se um lugar comum em ambientes corporativos.

O uso de tecnologias computacionais parece ser natural como instrumento de aprendizagem mas nem sempre é direto. Ter sua formação em uma graduação presencial que tenha implantado os $20 \%$, consentirá que, ao mesmo tempo que vivenciem a experiência acadêmica em uma potencialidade da aprendizagem mediada por computadores.

Com analogia aos aspectos legais, é preciso definir se os $20 \%$ em cada disciplina ou através de disciplinas completamente a distância, desde que respeitando os limites da legislação quanto a avaliação e estágios obrigatórios. Estas definições.

Com relação ao suporte técnico, é imprescindível que a equipe esteja atenta aos aspectos operacionais que garantirão o suporte confiável. Deve também fazer parte das atribuições dos responsáveis técnicos estarem atentos a possíveis atualizações, tanto de um mesmo sistema para correção de erros, quanto atualizações através de novos módulos.

Mais uma vez reforça-se que a conhecimento de pesquisa efetiva, os fundamentais resultado das avaliações apontaram para a importância de planejar e implantar um programa de capacitação docente e formatos e metodologias possíveis para capacitação, ótimos resultados foram obtidos quando se permitiu ao docente experimentar o papel de aluno EAD. Quanto ao acompanhamento, recomenda-se que sejam realizadas assessorias pedagógicas.

\section{REFERÊNCIAS}

BRASIL. Ministério da Educação - Secretaria de Educação Superior. Portaria do MEC/SESu nº ${ }^{\circ}$ 4.059, de 10 de dezembro de 2004. Regulamenta o artigo 81 da LDB 9.394/96 e revoga Portaria $n^{\circ}$. 2.253/2001. Disponível em: http://meclegis.mec.gov.br/documento/view/id/89.

BRASIL. Ministério da Educação - Secretaria de Educação Superior. Portaria do MEC/SESu nº ${ }^{\circ}$ 4.361, de 29 de dezembro de 2004. Normatizar os procedimentos de credenciamento de instituições para a oferta de cursos de graduação e educação profissional tecnológica à distância e revoga a Portaria Ministerial $n^{\circ} .301$ de 1998. Disponível em: <http://www.niltonlins.br/planejamento/Arquivos $\% 5$ CLegislacao\%5CPortariasMEC\%5CPO RTARIA_4361_12_2004.pdf>.

BRASIL. Ministério da Educação. Portaria do MEC $n^{\circ}$. 10, de 02 de julho de 2009. Fixa critérios para dispensa de avaliação in loco e dá outras providências. Disponível em: < http://portal.mec.gov.br/dmdocuments/portaria10_see d.pdf $>$.

BRASIL. Presidência da República - Casa Civil. Decreto $n^{\circ}$. 5.622, de 19 de Dezembro de 2005. Disponível em: http://portal.mec.gov.br/seed/arquivos/pdf/dec_5622.

BRASIL. Presidência da República - Casa Civil. Decreto $n^{\circ}$. 5.773, de 09 de maio de 2006.

Disponível em <

http://portal.mec.gov.br/seed/arquivos/pdf/legislacao/ decreton57731.pdf $>$.

PROGRAMA NACIONAL DE EDUCAÇÃO. Brasil. Disponível em: http:// portal.mec.gov.br/arquivos/pdf/pne.pdf.

AHAD, Ana Maria Abdul; ROCHA, Anderson Jacob; COSTA, Allan Thalles Galdino. • Inserção da EAD no ensino superior da cidade de Passos: uma experiência vanguardista. REVISTA CIÊNCIA ET PRAXIS, v. 8, n. 15, p. 13-18, 2017.

ARAÚJO, Laércio Ferreira. Democratização do Ensino Superior por meio das novas tecnologias da educação a distância: Uma revisão integrativa. Revista Aprendizagem em EAD, v. 3, n. 1, 2014.

CAETANO, Rodrigo da costa; RIBEIRO, Rosiane Lúcia; DE OLIVEIRA, Fabio Machado. EDUCAÇÃO A DISTÂNCIA E FORMAÇÂO DOCENTE: CONTRIBUIÇÕES E POSSIBILIDADES NA ERA TECNOLÓGICA. InterSciencePlace, v. 11, n. 3, 2016. 
CARVALHO, Nayara. Avaliação Institucional na Educação a Distância: um estudo de caso. Revista Meta: Avaliação, v. 9, n. 26, p. 255-271, 2017.

CERDAS, Luciene et al. Educação a distância e políticas de formação de professores: um balanço de estudos da área da educação. III Colóquio LusoBrasileiro de Educação a Distância e Elearning, p. 1$18,2013$.

FRANZIN, Sergio Francisco Loss et al. A INSTITUCIONALIZAÇÃO DA EAD E OS INDICADORES DE EFICÁCIA NA REDE FEDERAL DE EDUCAÇÃO, CIÊNCIA E TECNOLOGIA. InterSciencePlace, v. 1, n. 30, 2015.

JUNIOR, Emílio Rodrigues; FERNANDES, Fabricio Juliano. Proposta de inclusão de carga horária semipresencial em cursos superiores presenciais. Avaliação: Revista da Avaliação da Educação Superior, v. 19, n. 1, 2014.

JUNIOR, Klaus Schlünzen. Educação a distância no Brasil: caminhos, políticas e perspectivas. Educação Temática Digital, v. 10, n. 2, p. 16, 2009.

LIMA, Valeria Sperduti et al. FORMAÇÃO DA TUTORIA VIRTUAL A PARTIR DA INVESTIGAÇÃO DOS SUJEITOS. SIED: EnPEDSimpósio Internacional de Educação a Distância e Encontro de Pesquisadores em Educação a Distância, 2014.

LINO, Sônia Regina Lamego et al. Diretrizes para a institucionalização da gestão do conhecimento na rede federal de educação profissional, científica e tecnológica, Brasil. 2013.

LUVIZOTTO, Caroline Kraus; CARNIEL, Fabiane. A educação a distância na sociedade da informação e o processo de comunicação na sala de aulavirtual. Coleção PROPG Digital (UNESP), 2014.

MAIA, Gabrielle. Pressupostos pedagógicos da Educação a Distância: conhecendo as bases. Revista EDaPECI, v. 17, n. 1, p. 28-37, 2017.

MANGAN, Patrícia Kayser Vargas; ORTH, Miguel; DIAS, Maria Lúcia. Estratégias institucionais para a implementação dos $20 \%$ de EAD dos cursos de graduação a distância. Colabor@-A Revista Digital da CVA-RICESU, v. 7, n. 26, 2013.

MARCUZZO, Marlei Maria Veduim et al. EDUCAÇÃO A DISTÂNCIA: CONSTRUÇÕES QUE CONTRIBUEM PARA A SATISFAÇÃO DO ALUNO NA UNIVERSIDADE FEDERAL DE
SANTA MARIA. Revista Competência, v. 10, n. 1, p. 105-124, 2017.

MENESES, Yúla Pires da Silveira Fontenele et al. Estratégias Pedagógicas na Gestão de Ensino dos Cursos em EAD no UNINOVAFAPI. Revista Interdisciplinar, v. 9, n. 3, p. 143-148, 2016.

NASCIMENTO, E. S.; TEIXEIRA, V. M. M. N. Redação técnico-científica e pesquisa bibliográfica: algumas reflexões. Enfermagem Revista, v. 15, n. 2, p. 213-228, 2012.

OLIVEIRA, Hélio Carlos Miranda de. Perspectivas para a educação a distância no Brasil: referenciais de qualidade, releituras e trajetórias. Revista Eletrônica de Educação, v. 4, n. 2, p. 225-240, 2010.

RIBEIRO, Letícia Mendonça Lopes; DE SOUZA, Silvana Santos. A INCLUSÃO DE DISCIPLINAS A DISTÂNCIA NOS CURSOS DE GRADUAÇÃO PRESENCIAL: O ESTUDO DE CASO DOS ACADÊMICOS DA FACULDADE META. SIED: EnPED-Simpósio Internacional de Educação a Distância e Encontro de Pesquisadores em Educação a Distância, 2016.

SÁ, Georgina marafante. Educação a distância em instituições de ensino superior federais: aproximações e distanciamentos para a institucionalização. 2015.

SEBASTIÃO, Ana Paula Ferreira. A utilização do Ambiente Virtual de Aprendizagem Moodle em uma instituição de ensino superior pública. Revista Profissão Docente, v. 15, n. 32, 2015.

SEGENREICH, Stella Cecilia Duarte. A invasão silenciosa da EAD nos cursos de graduação presenciais no Brasil: questões de gestão e avaliação. Cadernos ANPAE, v. 1, p. 1-15, 2014.

SIMÕES, Cassiano. 20 ANOS DE AVALIAÇÃO DA APRENDIZAGEM EM EAD: ALGUMAS QUESTÕES ÉTICAS E NORMATIVAS. SIED: EnPED-Simpósio Internacional de Educação a Distância e Encontro de Pesquisadores em Educação a Distância, 2016.

Wellington Fernando da Silva Ferreira
Enfermeiro, Especialista em Saúde do Idoso e
Gerontologia.


Elia Machado de Oliveira

Enfermeira, Mestre em Cirurgia; Especialista em

Assistência de Enfermagem ao Paciente em Estado

Crítico, Especialista em Comunicação

Organizacional, Especialista em Auditoria de

Serviços de Saúde. 